\title{
Wave turbulence in magnetized plasmas
}

\author{
S. Galtier ${ }^{1,2}$ \\ ${ }^{1}$ Institut d'Astrophysique Spatiale, bâtiment 121, Université Paris-Sud XI, UMR 8617, 91405 Orsay, France \\ ${ }^{2}$ Institut Universitaire de France, France
}

Received: 7 October 2008 - Revised: 29 January 2009 - Accepted: 29 January 2009 - Published: 16 February 2009

\begin{abstract}
The paper reviews the recent progress on wave turbulence for magnetized plasmas (MHD, Hall MHD and electron MHD) in the incompressible and compressible cases. The emphasis is made on homogeneous and anisotropic turbulence which usually provides the best theoretical framework to investigate space and laboratory plasmas. The solar wind and the coronal heating problems are presented as two examples of application of anisotropic wave turbulence. The most important results of wave turbulence are reported and discussed in the context of natural and simulated magnetized plasmas. Important issues and possible spurious interpretations are also discussed.
\end{abstract}

\section{Introduction}

"The statistical evolution of interacting dispersive waves presents a solvable problem and is free of the closure difficulties associated with the theory of turbulence." - Benney and Newell (1967).

Wave turbulence is the study of the long-time statistical behavior of a sea of weakly nonlinear dispersive waves (Zakharov et al., 1992; Newell et al., 2001). The energy transfer between waves occurs mostly among resonant sets of waves and the resulting energy distribution, far from a thermodynamic equilibrium, is characterized by a wide power law spectrum and a high Reynolds number. This range of wavenumbers - the inertial range - is generally localized between large scales at which energy is injected in the system (sources) and small scales at which waves break or dissipate (sinks). Pioneering works on wave turbulence date back to the sixties when it was established that the stochastic initial value problem for weakly coupled wave systems has a natural asymptotic closure induced by the dispersive nature

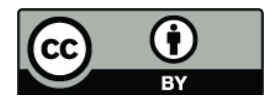

Correspondence to: S. Galtier (sebastien.galtier@ias.fr) of the waves and the large separation of linear and nonlinear time scales (Benney and Saffman, 1966; Benney and Newell, 1967, 1969). In the meantime, Zakharov and Filonenko (1966) showed that the wave kinetic equations derived from the wave turbulence analysis (with a Gaussian Ansatz applied to the four-point correlations of the wave amplitude) have exact equilibrium solutions which are the thermodynamic zero flux solutions but also - and more importantly finite flux solutions which describe the transfer of conserved quantities between sources and sinks. The solutions, first published for isotropic turbulence (Zakharov and Filonenko, 1966; Zakharov, 1967) were then extended to anisotropic turbulence (Kuznetsov, 1972).

Wave turbulence is a very common natural phenomenon with applications, for example, in capillary waves (Kolmakov et al., 2004; Abdurakhimov et al., 2008), gravity waves (Falcon et al., 2007), superfluid helium and processes of Bose-Einstein condensation (Kolmakov et al., 1995; Lvov et al., 2003), nonlinear optics (Dyachenko et al., 1992), inertial waves (Galtier, 2003; Morize et al., 2005) or Alfvén waves (Galtier et al., 2000; Kuznetsov, 2001; Chandran, 2005). The most important difference between plasmas and incompressible neutral fluids is the plethora of linear waves supported by the former. The direct consequence is that in weakly nonlinear plasmas the fundamental entities are waves rather than the eddies of strong turbulence (Kolmogorov, 1941; Krommes, 2002). Anisotropic turbulence is particularly well adapted to describe natural magnetized plasmas since a magnetic field is often present on the largest scale of the system, like in the inner interplanetary medium where the magnetic field lines form an Archimedean spiral near the equatorial plane (Goldstein and Roberts, 1999), at the solar surface where coronal loops and open magnetic flux tubes are found (Cranmer et al., 2007) or in planetary magnetospheres where shocks are found (Sahraoui et al., 2006).

In the present paper, a review is made on wave turbulence for magnetized plasmas which are described by the magnetohydrodynamics (MHD) approximation in the incompressible

Published by Copernicus Publications on behalf of the European Geosciences Union and the American Geophysical Union. 
and compressible case. The role played by the Hall term is discussed through the Hall MHD description as well as its small-scale limit of electron MHD. Physical motivations for developing wave turbulence theories are given in Sect. 2 where we first describe multiscale solar wind turbulence, and then present the coronal heating problem. In Sect. 4, the wave turbulence formalism is exposed with the basic ideas to derive the wave kinetic equations. Section 5 deals with the results obtained under different approximations (MHD, Hall MHD and electron MHD) in the incompressible and compressible case. Finally we conclude with a discussion in the last Section.

\section{Waves and turbulence in magnetized plasmas}

Waves and turbulence are ubiquitous in astrophysical plasmas. Their signatures are found in the Earth's magnetosphere (Sahraoui et al., 2006), the solar corona (Chae et al., 1998), the solar wind (Bruno and Carbone, 2005) or the interstellar medium (Elmegreen and Scalo, 2004; Scalo and Elmegreen, 2004). These regions are characterized by extremely large (magnetic) Reynolds numbers, up to $10^{13}$, with a range of available scales from $10^{18} \mathrm{~m}$ to a few meters.

\subsection{Multiscale solar wind}

Extensive investigations are made, in particular, in the interplanetary medium (and in the Earth's magnetosphere which is not the subject discussed here) where many in situ spacecrafts measurements are available. The solar wind plasma is found to be in a highly turbulent state with magnetic and velocity fluctuations detected from $10^{-6} \mathrm{~Hz}$ up to several hundred Hz (Coleman, 1968; Roberts et al., 1987; Leamon et al., 1998; Smith et al., 2006). The turbulent state of the solar wind was first suggested in 1968 (Coleman, 1968) when a power law behavior was reported for energy spectra with spectral indices lying between -1 and -2 (with the use of the Taylor "frozen-in flow" hypothesis). More precise measurements revealed that the spectral index at low frequency $(<1 \mathrm{~Hz})$ is often about -1.7 which is closer to the Kolmogorov prediction (Kolmogorov, 1941) for neutral fluids $(-5 / 3)$ rather than the Iroshnikov-Kraichnan prediction (Iroshnikov, 1964; Kraichnan, 1965) for magnetized fluids $(-3 / 2)$. Alfvén waves are also well observed since 1971 (Belcher and Davis, 1971) with a strong domination of antisunward propagative waves at short heliocentric distances (less than $1 \mathrm{AU}$ ). Since pure (plane) Alfvén waves are exact solutions of the ideal incompressible MHD equations (see e.g., Pouquet, 1993), nonlinear interactions should be suppressed if only one type of waves is present. Therefore sunward Alfvén waves, although subdominant, play an important role in the internal solar wind dynamics.

The variance analysis of the magnetic field components and of its magnitude shows clearly that the magnetic field vector of the (polar) solar wind has a varying direction but with only a weak variation in magnitude (Forsyth et al., 1996). Typical values give a normalized variance of the field magnitude smaller than $10 \%$ whereas for the components it can be as large as $50 \%$. In these respects, the inner interplanetary magnetic field may be seen as a vector lying approximately around an Archimedean spiral direction with only weak magnitude variations (Barnes, 1981). Solar wind anisotropy with more power perpendicular to the mean magnetic field than that parallel to it, is pointed out by data analysis (Klein et al., 1993) that provides a ratio of power up to 30 . From single-point spacecraft measurements it is however not possible to specify the exact threedimensional form of the spectral tensor of the magnetic or velocity fluctuations. In the absence of such data, a quasi two-dimensional model was proposed (Bieber et al., 1996) in which wave vectors are nearly perpendicular to the largescale magnetic field. It is found that about $85 \%$ of solar wind turbulence possesses a dominant 2-D component. Additionally, solar wind anisotropies is detected through radio wave scintillations which reveal that density spectra close to the Sun are highly anisotropic with irregularities stretched out mainly along the radial direction (Armstrong et al., 1990).

For frequencies larger than $1 \mathrm{~Hz}$, a steepening of the magnetic fluctuation power law spectra is observed over more than two decades (Coroniti et al., 1982; Denskat et al., 1983; Leamon et al., 1998; Bale et al., 2005; Smith et al., 2006) with a spectral index close to -3 . This new inertial range seems to be characterized by a bias of the polarization suggesting that these fluctuations are likely to be right-hand polarized, outward propagating waves (Goldstein et al., 1994). Various indirect lines of evidence indicate that these waves propagate at large angles to the background magnetic field and that the power in fluctuations parallel to the background magnetic field is still less than the perpendicular one (Coroniti et al., 1982; Leamon et al., 1998). For these reasons, it is thought (Stawicki et al., 2001) that Alfvén - left circularly polarized - fluctuations are suppressed by proton cyclotron damping and that the high frequency power law spectra are likely to consist of whistler waves. This scenario is supported by multi-dimensional direct numerical simulations of compressible Hall MHD turbulence in the presence of an ambient field (Ghosh et al., 1996) where a steepening of the spectra was found on a narrow range of wavenumbers, and associated with the appearance of right circularly polarized fluctuations. This result has been recently confirmed numerically with a turbulent cascade model (shell model) based on 3D Hall MHD in which a well extended steeper power law spectrum was found at scale smaller than the ion skin depth (Galtier and Buchlin, 2007). (Note that in this cascade model no mean magnetic field is assumed.) However, the exact origin of the change of statistical behavior is still under debate (Markovskii et al., 2008): for example, an origin from compressible effects is possible in the context of Hall MHD (Alexandrova et al., 2008); a kinetic description was 
also proposed (Howes et al., 2008).

The solar wind plasma is currently the subject of a new extensive research around the origin of the spectral break observed in the magnetic fluctuations. We will see that wave turbulence may have a central role in the sense that it is a useful point of departure for understanding the detailed physics of solar wind turbulence. In particular, it gives strong results in regards to the possible multiscale behavior of magnetized plasmas as well as the intensity of the anisotropic transfer between modes.

\subsection{Heating of the solar corona}

Although it is not easy to measure directly the coronal magnetic field, it is now commonly accepted that the structure of the low solar corona is mainly due to the magnetic field (Aschwanden et al., 2001). The high level of coronal activity appears through a perpetual impulsive reorganization of magnetic structures over a large range of scales, from about $10^{5} \mathrm{~km}$ until the limit of resolution, about one arcsec $(<726 \mathrm{~km})$. The origin of the coronal reorganization is currently widely studied in solar physics. Information about the solar corona comes from spacecraft missions like SoHO or TRACE launched in the 1990s, or from the new spacecraft STEREO and Hinode. The most recent observations reveal that coronal loops are not yet resolved transversely and have to be seen as tubes made of a set of strands which radiate alternatively. In fact, it is very likely that structures at much smaller scales exist but have not yet been detected (see e.g., Warren, 2006).

Observations in UV and X-ray show a solar corona extremely hot with temperatures exceeding $10^{6} \mathrm{~K}$ - close to hundred times the solar surface temperature. These coronal temperatures are highly inhomogeneous: in the quiet corona much of the plasma lies near $1-2 \times 10^{6} \mathrm{~K}$ and $1-8 \times 10^{6} \mathrm{~K}$ in active regions. Then, one of the major questions in solar physics concerns the origin of such high values of coronal temperature. The energy available in the photosphere characterized by granules - is clearly sufficient to supply the total coronal losses (Priest, 1982) which is estimated to be $10^{4} \mathrm{~J} \mathrm{~m}^{-2} \mathrm{~s}^{-1}$ for active regions and about one or two orders of magnitude smaller for the quiet corona and coronal holes where open magnetic field lines emerge. The main issue is thus to understand how the available photospheric energy is transferred and accumulated in the solar corona, and by what processes it is dissipated.

In active region loops, analyses made by spectrometers show that the plasma velocity can reach values up to $50 \mathrm{~km} / \mathrm{s}$ (Brekke et al., 1997). The highly dynamical nature of some coronal loops is also pointed out by non-thermal velocities reaching sometimes $50 \mathrm{~km} / \mathrm{s}$ as it was revealed for example by SoHO (Chae et al., 1998). These observations give also evidences that the line broadening is due to motions which are still not resolved neither in space, with scales smaller than the diameter of coronal loops, nor in time, with timescales shorter than the exposure time of the order of few seconds. These velocity measurements are very often interpreted as a signature of MHD turbulence where small scales are produced naturally via a nonlinear cascade of energy. In the light of the most recent observations, it seems fundamental to study, both theoretically and numerically, the impact of small-scale phenomena on the coronal heating. Note that the most recent Hinode pictures seem to show a magnetic field controlled by plasma turbulence at all scales in which Alfvén waves are omnipresent (see e.g., Doschek et al., 2007; Nishizuka et al., 2008). Thus, the turbulent activity of the corona is one of the key issues to understand the heating processes.

In the framework of turbulence, the energy supplied by the photospheric motions and transported by Alfvén waves through the corona is transferred towards smaller and smaller scales by nonlinear coupling between modes (the so-called energy cascade) until dissipative scales are reached from which the energy is converted into heating. The main coronal structures considered in such a scenario are the magnetic loops which cover the solar surface. Each loop is basically an anisotropic bipolar structure anchored in the photosphere. It forms a tube of magnetic fields in which the dense and hot matter is confined. Because a strong guiding magnetic field $\left(\mathbf{B}_{\mathbf{0}}\right)$ is present, the nonlinear cascade that occurs is strongly anisotropic with small scales mainly developed in the $\mathbf{B}_{\mathbf{0}}$ transverse planes. Most of the models published deals with isotropic MHD turbulence (see e.g., Hendrix and Van Hoven, 1996) and it is only very recently that anisotropy has been included in turbulent heating models (Buchlin and Velli, 2007).

The latest observations show that waves and turbulence are among the main ingredients of the solar coronal activity. Weak MHD turbulence is now invoked has a possible regime for some coronal loops since a very small ratio is found between the fluctuating magnetic field and the axial component (Rappazzo et al., 2007, 2008). Inspired by the observations and by recent direct numerical simulations of 3-D MHD turbulence (Bigot et al., 2008b), an analytical model of coronal structures has been proposed (Bigot et al., 2008c) where the heating is seen as the end product of a wave turbulent cascade. Surprisingly, the heating rate found is non negligible and may explain the observational predictions.

The coronal heating problem also concerns the regions where the fast solar wind is produced, i.e. the coronal holes (Hollweg and Isenberg, 2002; Cranmer et al., 2007). Observations seem to show that the heating affects preferentially the ions in the direction perpendicular to the mean magnetic field. The electrons are much cooler than the ions, with temperatures generally less than or close to $10^{6} \mathrm{~K}$ (see e.g., David et al., 1998). Additionally, the heavy ions become hotter than the protons within a solar radius of the coronal base. Ion cyclotron waves could be the agent which heats the coronal ions and accelerates the fast wind. Naturally the question of the origin of these high frequency waves arises. 
Among different scenarios, turbulence appears to be a natural and efficient mechanism to produce ion cyclotron waves. In this case, the Alfvén waves launched at low altitude with frequencies in the MHD range, would develop a turbulent cascade to finally degenerate and produce ion cyclotron waves at much higher frequencies. In that context, the wave turbulence regime was considered in the weakly compressible MHD case at low- $\beta$ plasmas (where $\beta$ is the ratio between the thermal and magnetic pressure) in order to analyze the nonlinear three-wave interaction transfer to high frequency waves (Chandran, 2005). The wave turbulence calculation shows - in absence of slow magnetosonic waves - that MHD turbulence is a promising explanation for the anisotropic ion heating.

\section{Fully developed - wave and strong - turbulence}

This review paper is devoted to wave turbulence. Therefore, it is important to stress the difference with the so-called strong turbulence. In this section, we will also see how the theoretical questions addressed at the end of the 20th century have led to the emergence of a large number of papers on wave turbulence in magnetized plasmas and to many efforts to characterize the fundamental role of anisotropy.

\subsection{Navier-Stokes turbulence}

Navier-Stokes turbulence is basically a strong turbulent problem in which it is impossible to perform (a non trivial and) a consistent linearization of the equations against a stationary homogeneous background. We remind that wave turbulence demands the existence of linear (dispersive) propagative waves as well as a large separation of linear and nonlinear (eddy-turnover) time scales (see e.g., Benney and Newell, 1969). In his third 1941 turbulence paper, Kolmogorov (1941) found that an exact and nontrivial relation may be derived from Navier-Stokes equations for the thirdorder longitudinal structure function (Kolmogorov, 1941). Because of the rarity of such results, the Kolmogorov's fourfifth's law is considered as one of the most important results in turbulence (Frisch, 1995). Basically, the four-fifth's theorem makes the following link in the physical space between a two-point measurement, separated by a distance $\mathbf{r}$, and the distance itself (in 3-D):

$$
-\frac{4}{5} \varepsilon^{v} r=\left\langle\left(v_{\|}^{\prime}-v_{\|}\right)^{3}\right\rangle,
$$

where \langle\rangle denotes an ensemble average, the parallel direction $\|$ is the one along the vector separation $\mathbf{r}, v$ is the velocity and $\varepsilon^{v}$ is the mean (kinetic) energy injection, transfer and dissipation rate per unit mass. To obtain this exact result, the assumptions of homogeneity and isotropy are made (Batchelor, 1953). The former assumption is satisfied as long as we are at the heart of the fluid (far from the boundaries) and the latter is also satisfied if no external agent (like, for example, rotation or stratification) is present. Additionally, we need to consider the long time limit for which a stationary state is reached with a finite $\varepsilon^{v}$ and we take the infinite Reynolds number limit (i.e. the viscosity $\nu \rightarrow 0$ ) for which the mean energy dissipation rate per unit mass tends to a finite positive limit. Therefore, the exact prediction is valid in a wide inertial range. This prediction is well supported by the experimental data (see e.g., Frisch, 1995).

The four-fifth's law is a fundamental result used to develop heuristic spectral scaling laws like the famous - but not exact $-5 / 3$-Kolmogorov energy spectrum. This point makes a fundamental difference with wave turbulence where the power law spectra found are exact solutions of the asymptotically exact wave turbulence equations. Nevertheless, the term "Kolmogorov theory" is often associated to the $-5 / 3$ spectrum since there exists a theory behind in the physical space.

\subsection{Incompressible MHD turbulence}

\subsubsection{Strong turbulence}

In the MHD case, wave turbulence is possible. The main reason is that Alfvén waves are linear solutions when a stationary homogeneous background magnetic field $\mathbf{B}_{\mathbf{0}}$ is applied. These statements seem to be obvious for incompressible MHD but we will see that the problem is more subtle and the existence of a wave turbulence theory was the object of many discussions basically because those waves are only pseudo-dispersive (i.e., the frequency $\omega$ is proportional to $k$ ).

The question of the existence of an exact relation between a two-point measurement, separated by a distance $\mathbf{r}$, and the distance itself is naturally addressed for strong (without $\mathbf{B}_{\mathbf{0}}$ ) turbulence. A positive answer was given by Politano \& Pouquet only in 1998 (see also, Chandrasekhar, 1951) for incompressible MHD turbulence. The presence of the magnetic field and its coupling with the velocity field renders the problem more difficult and, in practice, we are dealing with a couple of equations. In this case, the possible formulation in 3-D is:

$$
-\frac{4}{3} \varepsilon^{ \pm} r=\left\langle\left(z_{\|}^{\prime-}-z_{\|}^{-}\right) \sum_{i}\left(z_{i}^{+}-z_{i}^{+}\right)^{2}\right\rangle,
$$

where the parallel direction $\|$ is still the one along the vector separation $\mathbf{r}, \mathbf{z}^{ \pm}=\mathbf{v} \pm \mathbf{b}$ is the Elsässer fields (with $\mathbf{b}$ normalized to a velocity field) and $\varepsilon^{ \pm}$is the mean energy dissipation rate per unit mass associated to the Elsässer energies. To obtain these exact results, the assumptions of homogeneity and isotropy are still made, and we also consider the long time limit for which a stationary state is reached with a finite $\varepsilon^{ \pm}$ and we take the infinite kinetic and magnetic Reynolds number limit ( $\nu \rightarrow 0$ and the magnetic diffusivity $\eta \rightarrow 0$ ) for which the mean energy dissipation rates per unit mass have a finite positive limits. Therefore, the exact prediction is again valid, at first order, in a wide inertial range. This prediction is still 
widely used in the literature to analyze space plasma data (see e.g., Sorriso-Valvo et al., 2007; MacBride et al., 2008).

\subsubsection{Iroshnikov-Kraichnan spectrum}

The isotropy assumption used to derive the 4/3's law, which mainly appears in the development of the kinematics (Batchelor, 1953), is stronger for magnetized than neutral fluids since most of the situations encountered in astrophysics are far from isotropy where a $\mathbf{B}_{\mathbf{0}}$ magnetic field is often present (see Sect. 2). Although this law is a fundamental result which may be used to develop a heuristic spectral scaling law, the role of $B_{0}$ has to be clarified. Indeed, we have now two timescales: the eddy-turnover time and the Alfvén time. The former is similar to the eddy-turnover time in Navier-Stokes turbulence and may be associated to the distortion of wave packets, whereas the latter may be seen as the duration of interaction between two counter-propagating linear plane Alfvén waves (see Fig. 1).

During a collision, there is a deformation of the wave packets in such a way that energy is transferred mainly at smaller scales. The multiplicity of collisions leads to the formation of a well extended power law energy spectrum whose index lies between $-5 / 3$ (Kolmogorov prediction) and $-3 / 2$ (Iroshnikov-Kraichnan prediction) according to the phenomenology used, i.e. with or without the Alfvén wave effect. Note that in the Iroshnikov approach the presence of a strong magnetic field is explicitly assumed whereas it is not in the Kraichnan's one. In the latter case, it is claimed that the small-scale fluctuations see the large-scales - in the sub-inertial range - as a spatially uniform magnetic field. It is important to note that the exact isotropic prediction in physical space, Eq. (2), corresponds dimensionally to a $-5 / 3$ energy spectrum. It is therefore less justified to use the term "theory" for the Iroshnikov-Kraichnan spectrum than for the Kolmogorov one in Navier-Stokes flows.

\subsubsection{Breakdown of isotropy}

The weakness of the Iroshnikov-Kraichnan phenomenology is the apparent contradiction between the (in)direct presence of a strong uniform magnetic field and the assumption of isotropy. One of the most important difference between neutral and magnetized fluids is the impossibility in the latter case to remove a large-scale (magnetic) field by a galilean transform. The role of a uniform magnetic field has been widely discussed in the literature and, in particular, during the last decades (see e.g., Montgomery and Turner, 1981; Shebalin et al., 1983; Matthaeus et al., 1996; $\mathrm{Ng}$ and Bhattacharjee, 1996; Verma, 2004). At strong $\mathbf{B}_{\mathbf{0}}$ intensity, one of the most clearly established results is the bi-dimensionalization of MHD turbulent flows with a strong reduction of nonlinear transfers along $\mathbf{B}_{\mathbf{0}}$. The consequence is an energy concentration near the plane $\mathbf{k} \cdot \mathbf{B}_{\mathbf{0}}=0$, a result il-

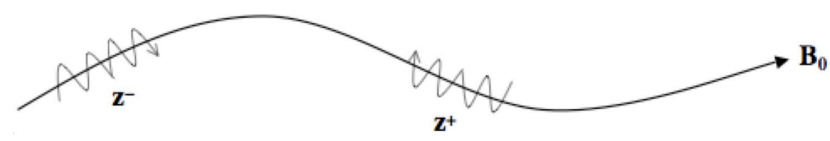

Fig. 1. Schematic Alfvén wave packets propagating along a magnetic field line.

lustrated later on by direct numerical simulations in two and three space dimensions (Shebalin et al., 1983).

The effects of a strong uniform magnetic field may be handled through an analysis of resonant triadic interactions (Shebalin et al., 1983) between the wavevectors $(\mathbf{k}, \mathbf{p}, \mathbf{q})$ which satisfy the relation

$\mathbf{k}=\mathbf{p}+\mathbf{q}$,

whereas the associated wave frequencies satisfy

$\omega(\mathbf{k})=\omega(\mathbf{p})+\omega(\mathbf{q})$.

For incompressible MHD, the Alfvén frequency is

$\omega(\mathbf{k})= \pm \mathbf{k} \cdot \mathbf{B}_{\mathbf{0}}= \pm k_{\|} B_{0}$,

where $\|$ defines the direction along $\mathbf{B}_{\mathbf{0}}(\perp$ will be the perpendicular direction to $\mathbf{B}_{\mathbf{0}}$ taken in velocity unit). The solution of these three-wave resonant conditions gives for example, $q_{\|}=0$, which implies a spectral transfer only in the perpendicular direction. For a strength of $B_{0}$ well above the r.m.s. level of the kinetic and magnetic fluctuations, the nonlinear interactions of Alfvén waves may dominate the dynamics of the MHD flow leading to the regime of wave turbulence (see Sect. 4) where the energy transfer, stemming from three-wave resonant interactions, can only increase the perpendicular component of the wavevectors, while the nonlinear transfers is completely inhibited along $\mathbf{B}_{\mathbf{0}}$. The end result is a strongly anisotropic flow.

\subsubsection{Emergence of anisotropic laws}

Another important issue discussed in the literature is the relationship between perpendicular and parallel scales in anisotropic MHD turbulence (see e.g., Higdon, 1984; Goldreich and Sridhar, 1995; Boldyrev, 2006). In order to take into account anisotropy, Goldreich and Sridhar (1995) proposed a heuristic model based on a critical balance between the Alfvén time and the eddy-turnover time scale, respectively

$\tau_{A} \sim \ell_{\|} / B_{0}$

and

$\tau_{\text {eddy }} \sim \ell_{\perp} / u_{\ell}$,

where $\ell_{\|}$and $\ell_{\perp}$ are typical length scales parallel and perpendicular to $\mathbf{B}_{\mathbf{0}}$, and with $\tau_{A}=\tau_{\text {eddy }}$ at all inertial scales. The 
latter relation leads trivially to $u_{\ell} \sim B_{0} \ell_{\perp} / \ell_{\|}$. Following the Kolmogorov arguments, one ends up with a

$E\left(k_{\perp}, k_{\|}\right) \sim k_{\perp}^{-5 / 3}$

energy spectrum (where $\mathbf{k} \equiv\left(\mathbf{k}_{\perp}, k_{\|}\right)$and $\left.k_{\perp} \equiv\left|\mathbf{k}_{\perp}\right|\right)$, and with the anisotropic scaling law (with $u_{\ell}^{2} / \tau_{\text {eddy }}=$ constant)

$k_{\|} \sim k_{\perp}^{2 / 3}$

This heuristic prediction means that anisotropy is stronger at smaller scales.

In the same spirit, a generalization of this result has been proposed in an attempt to model MHD flows in both the weak and strong turbulent regimes, as well as in the transition between them (Galtier et al., 2005). In this heuristic model, the time-scale ratio $\chi=\tau_{A} / \tau_{\text {eddy }}$ is supposed to be constant at all inertial scales but not necessarily equal to unity. The relaxation of this constraint enables to still recover the anisotropic scaling law Eq. (9) which now includes $B_{0}$,

$k_{\|} \sim k_{\perp}^{2 / 3} / B_{0}$

and to find a universal prediction for the total energy spectrum

$E\left(k_{\perp}, k_{\|}\right) \sim k_{\perp}^{-\alpha} k_{\|}^{-\beta}$, with $: 3 \alpha+2 \beta=7$.

According to direct numerical simulations (see e.g., Cho and Vishniac, 2000; Maron and Goldreich, 2001; Shaikh and Zank, 2007; Bigot et al., 2008b), the anisotropic scaling law between parallel and perpendicular scales Eq. (9) seems to be a robust result and an approximately constant ratio $\chi$, generally smaller than one, is found between the Alfvén and the eddy-turnover times. This sub-critical value of $\chi$ implies therefore a dynamics mainly driven by Alfvén waves interactions. Note that the presence of $B_{0}$ in relation Eq. (10) shows the convergence towards wave turbulence $\left(B_{0} \rightarrow+\infty\right.$, with respect to the fluctuations) for which the parallel transfer is totally frozen.

The question of the spectral indices is still a challenging problem in anisotropic turbulence (Sagaut and Cambon, 2008). The main conclusion of Bigot et al. (2008b) is that the difficulty to make their measurements is generally underestimated in a sense that the scaling prediction in $k_{\perp}$ may change significantly when $E\left(k_{\perp}, k_{\|}\right)$is plotted at a given $k_{\|}$instead of $E\left(k_{\perp}\right)$ : indeed, the 2-D state $E\left(k_{\perp}, k_{\|}=0\right)$ may play a singular role in the dynamics with a scaling in $k_{\perp}$ very different from the one given by the 3 -D modes $E\left(k_{\perp}, k_{\|}>0\right)$. This comment holds primary for direct numerical simulations where technically it is currently possible to make this distinction, observations being still far from this possibility. This point will be further discussed in the last Section. Note finally that all these spectral predictions suffer from rigorous justifications and the word "theory" that we find very often in the literature is not justified at all. A breakthrough could be achieved if one could develop the equivalent of an exact fourfifth's law for anisotropic MHD turbulence. Then, a dimensional derivation from this law could lead to an anisotropic spectral prediction and in some sense, for the first time, the possibility of having a theoretical link between strong and wave turbulence. To date, it is still an open issue.

\subsubsection{Towards a wave turbulence theory}

In view of the importance of anisotropy in natural magnetized plasma (see Sect. 2), Sridhar and Goldreich (1994) suggested that a plasma evolving in a medium permeated by a strong uniform magnetic field and in the regime of wave turbulence is characterized by four-wave nonlinear interactions. The essence of wave turbulence is the statistical study of large ensembles of weakly interacting waves via a systematic asymptotic expansion in powers of small nonlinearity. This technique leads finally to the exact derivation of wave kinetic equations for the invariants of the system like the energy spectrum (see Sect. 4). In MHD, it is the presence of a strong uniform magnetic field $\mathbf{B}_{\mathbf{0}}$ that allows to introduce a small parameter in the system, namely the ratio between the fluctuating fields and $B_{0}$. The results found by Sridhar and Goldreich in 1994 imply that the asymptotic development has no solution at the first order and that we need to go to the second order to describe wave turbulence in MHD. Several articles, at the level of the phenomenology, were published to contest this conclusion and sustain the non trivial character of the three-wave interactions (see e.g., Montgomery and Matthaeus, 1995; Ng and Bhattacharjee, 1996; Verma, 2004). In response, a detailed theory was finally given in 2000 (Galtier et al., 2000; Nazarenko et al., 2001; Galtier et al., 2002) and first signatures that may be attributed to wave turbulence were found by Perez and Boldyrev (2008) in numerical simulations of a reduced form of the MHD equations (Galtier and Chandran, 2006). The detection of the wave turbulence regime from direct numerical simulations of the original MHD equations is still a difficult task but recent progress has been made in which temporal, structural and spectral signatures are reported (Bigot et al., 2008a,b). Note that current efforts are also made to analyze the effects of other inviscid invariants, like the cross helicity, on the scaling laws of wave turbulence (Lithwick and Goldreich, 2003; Chandran, 2008).

\subsection{Wave turbulence in compressible MHD}

Most of the investigations devoted to wave turbulence refer to isotropic media where the well known conformal transform proposed by Zakharov and Filonenko (1966) may be applied to find the so-called Kolmogorov spectra (see Sect. 4.4). (It is interesting to note that a similar transform was used by Kraichnan to investigate the problem of 2-D turbulence (Kraichnan, 1967).) The effect of anisotropy in a plasma was studied to a smaller extent. The first example is given by 
magnetized ion-sound waves (Kuznetsov, 1972). The compressible MHD case was analyzed later by Kuznetsov (2001) for a situation where the plasma (thermal) pressure is small compared with the magnetic pressure (small $\beta$ limit). In this case, the main nonlinear interaction of MHD waves is the scattering of a fast magneto-acoustic and Alfvén waves on slow magneto-acoustic waves. In these processes, the fast and Alfvén waves act as high-frequency waves with respect to the slow waves. To simplify the analysis, other three-wave interaction processes that do not involve slow waves are neglected.

A variant of this wave turbulence analysis in compressible MHD was proposed by Chandran (2005) for small $\beta$ in which, to simplify the analysis, the slow waves are neglected and a constant density is imposed. The other (mathematical) difference is that in the former analysis the hamiltonian formalism was used whereas an eulerian description was employed in the latter case. The compressible case is much more difficult to analyze than the incompressible one and some simplifications are necessary. To date, no general theory - without simplification - has been reached for compressible MHD wave turbulence.

\subsection{Wave turbulence in Hall and electron MHD}

Modeling the physics of a plasma beyond the MHD approximation, namely on spatial scales shorter than the ion inertial length $d_{i}$ (but larger than the electron inertial length $d_{e}$ ) and time scales shorter than the ion cyclotron period $1 / \omega_{c i}$ is a highly studied topic. Note also the existence of the gyrokinetic approach for weakly collisional plasmas (see e.g., Schekochihin et al., 2008) in which time scales are supposed to be much larger than $1 / \omega_{c i}$. A simple model is the electron MHD approximation (Kingsep et al., 1990) in which one assumes that ions do not have time to follow electrons and provide a static homogeneous background on which electrons move. The electron MHD approximation is particularly relevant in the context of collisionless magnetic reconnection where the diffusion region develops multiscale structures corresponding to ion and electron characteristic lengths (Biskamp, 1997; Bhattacharjee, 2004; Yamada, 2007).

An important issue in electron MHD turbulence is about the role of whistler waves in the dynamics. Biskamp et al. (1999) argued that although whistler wave propagation effects are non negligible in electron MHD turbulence, the local spectral energy transfer process is independent of the linear wave dispersion relation and the energy spectrum may be predicted by a Kolmogorov type argument (Biskamp et al., 1996; $\mathrm{Ng}$ et al., 2003); this numerical analysis was made for an isotropic medium $\left(B_{0}=0\right)$. Dastgeer et al. (2000) investigated the turbulence regime in the presence of a moderate background magnetic field $B_{0}$, and provide convincing numerical evidence that turbulence may be anisotropic. They argue that although whistler waves may appear to play a negligible effect in determining the spectral index, they play an important role in setting up an anisotropic turbulent cascade. The whistler wave turbulence regime was then investigated theoretically by Galtier and Bhattacharjee $(2003,2005)$ in the limit $B_{0} \rightarrow+\infty$ (with respect to the fluctuations). It was shown that similarly to the MHD case, anisotropy is a central feature of such a turbulence. Attempts to find anisotropic law for electron MHD was made by Cho and Vishniac (2004) (see also, Galtier et al., 2005) and a scaling law in $k_{\|} \sim k_{\perp}^{1 / 3}$ was found both heuristically and numerically.

Hall MHD is an extension of the standard MHD where the ion inertia is retained in Ohm's law. It provides a multiscale description of magnetized plasmas from which both the standard and the electron MHD approximation may be recovered. Hall MHD is often used to understand, for example, the magnetic field evolution in neutron star crusts (Goldreich and Reisenegger, 1992), the turbulent dynamo (Mininni et al., 2003), the formation of filaments (Laveder, Passot and Sulem, 2002), the multiscale solar wind turbulence (Ghosh et al., 1996; Krishan and Mahajan, 2004; Galtier, 2006a,b), or the dynamics of the magnetosheath (Belmont and Rezeau, 2001). Anisotropy in Hall MHD is clearly less understood than in MHD or electron MHD mainly because the numerical treatment is more limited since a wide range of scales are necessary to detect any multiscale effects. From a theoretical point of view, it is only recently that a wave turbulence theory has been achieved for the incompressible case (Galtier, 2006b; Sahraoui et al., 2007). For such a turbulence, a global tendency towards anisotropy was found (with, however, a weaker effect at intermediate scales) with nonlinear transfers preferentially in the direction perpendicular to the external magnetic field $\mathbf{B}_{\mathbf{0}}$. The energy spectrum is characterized by two inertial ranges, which are exact solutions of the wave kinetic equations, separated by a knee. The position of the knee corresponds to the scale where the Hall term becomes sub/dominant. To date, the compressible case is still an open problem in Hall MHD wave turbulence. Note, however, the first step made by Sahraoui, Belmont and Rezeau (2003) to formulate the problem of weakly nonlinear compressible Hall MHD equations in terms of Hamiltonian system.

\section{Wave turbulence formalism}

\subsection{Wave amplitude equation}

Wave turbulence is the study of the long time statistical behavior of a sea of weakly nonlinear dispersive waves; this regime is described by the wave kinetic equations. In this section we present the wave turbulence formalism which leads to these nonlinear equations. We will use the inviscid model equation

$$
\frac{\partial \mathbf{u}(\mathbf{x}, t)}{\partial t}=\mathcal{L}(\mathbf{u})+\varepsilon \mathcal{N}(\mathbf{u}, \mathbf{u}),
$$


where $\mathbf{u}$ is a stationary random vector, $\mathcal{L}$ is a linear operator which insures that waves are solutions of the linear problem, and $\mathcal{N}$ is a quadratic nonlinear operator (like for MHD-type flows). The factor $\varepsilon$ is a small parameter $(0<\varepsilon \ll 1)$ which insures that the nonlinearities are weak. For all the applications considered here, the smallness of the nonlinearities is the result of the presence of a strong uniform magnetic field $\mathbf{B}_{\mathbf{0}}$; the operator $\mathcal{L}$ is thus proportional to $B_{0}$.

We introduce the 3-D direct and inverse Fourier transforms

$\mathbf{u}(\mathbf{x}, t)=\int_{\mathrm{R}^{3}} \mathbf{A}(\mathbf{k}, t) \exp (i \mathbf{k} \cdot \mathbf{x}) d \mathbf{k}$,

$\mathbf{A}(\mathbf{k}, t)=\frac{1}{(2 \pi)^{3}} \int_{\mathrm{R}^{3}} \mathbf{u}(\mathbf{x}, t) \exp (-i \mathbf{k} \cdot \mathbf{x}) d \mathbf{x}$.

Therefore, a Fourier transform of Eq. (12) gives for the $j$ component

$$
\begin{aligned}
& \left(\frac{\partial}{\partial t}+i \omega(\mathbf{k})\right) A_{j}(\mathbf{k}, t)= \\
& \quad \varepsilon \int_{\mathrm{R}^{6}} \mathcal{H}_{j m n}^{\mathbf{k p q}} A_{m}(\mathbf{p}, t) A_{n}(\mathbf{q}, t) \delta(\mathbf{k}-\mathbf{p}-\mathbf{q}) d \mathbf{p} d \mathbf{q},
\end{aligned}
$$

where $\omega(\mathbf{k})=\omega_{k}$ is given by the appropriate dispersion relation (with in general $\omega(-\mathbf{k})=-\omega(\mathbf{k}))$ and $\mathcal{H}$ is a symmetric function in its vector arguments which basically depends on the quadratic nonlinear operator $\mathcal{N}$. Note the use of the Einstein's notation. We introduce

$\mathbf{A}(\mathbf{k}, t)=\mathbf{a}(\mathbf{k}, t) e^{-i \omega_{k} t}$,

and obtain in the interaction representation

$\frac{\partial a_{j}(\mathbf{k})}{\partial t}=\varepsilon \int_{\mathrm{R}^{6}} \mathcal{H}_{j m n}^{\mathbf{k p q}} a_{m}(\mathbf{p}) a_{n}(\mathbf{q}) e^{i \Omega_{k, p q} t} \delta_{k, p q} d \mathbf{p} d \mathbf{q}$,

where the Dirac delta function $\delta_{k, p q}=\delta(\mathbf{k}-\mathbf{p}-\mathbf{q})$ and $\Omega_{k, p q}=\omega_{k}-\omega_{p}-\omega_{q}$; note that the time dependence in fields, a, is omitted for simplicity. Eq. (17) is a fundamental equation: it is the wave amplitude equation whose dependence in $\varepsilon$ means that weak nonlinearities will modify only slowly in time the wave amplitude. By nature, all the problems considered here (MHD, electron and Hall MHD) involve mainly three-wave interaction processes as it is expected by the form of the wave amplitude equation. The exponentially oscillating term is essential for the asymptotic closure: indeed, we are interested by the long time statistical behavior, i.e. a nonlinear transfer time much greater than the wave period. As a consequence, most of the nonlinear terms will be destroyed by random phase mixing and only a few of them - called the resonance terms - will survive. Note the necessity of having dispersive waves. Before going to the statistical formalism, we note the following general properties that will be used

$\mathcal{H}_{j m n}^{\mathbf{k p q}}=\left(\mathcal{H}_{j m n}^{-\mathbf{k}-\mathbf{p}-\mathbf{q}}\right)^{*}$,

$\mathcal{H}_{j m n}^{\mathbf{k p q}}$ is symmetric in $(\mathbf{p}, \mathbf{q})$ and $(m, n)$,

$\mathcal{H}_{j m n}^{0 \text { pq }}=0$,

where, ${ }^{*}$, stands for the complex conjugate.

\subsection{Statistics and asymptotics}

We turn now to a statistical description, introduce the ensemble average $\langle\ldots\rangle$ and define the density tensor for homogeneous turbulence

$q_{j j^{\prime}}\left(\mathbf{k}^{\prime}\right) \delta\left(\mathbf{k}+\mathbf{k}^{\prime}\right)=\left\langle a_{j}(\mathbf{k}) a_{j^{\prime}}\left(\mathbf{k}^{\prime}\right)\right\rangle$.

We also assume that on average $\langle\mathbf{u}(\mathbf{x}, t)\rangle=0$ which leads to the relation $\mathcal{H}_{j m n}^{0 \mathbf{p q}}=0$. From the nonlinear Eq. (17), we find

$$
\begin{gathered}
\frac{\partial q_{j j^{\prime}} \delta\left(\mathbf{k}+\mathbf{k}^{\prime}\right)}{\partial t}=\left\langle a_{j^{\prime}}\left(\mathbf{k}^{\prime}\right) \frac{\partial a_{j}(\mathbf{k})}{\partial t}\right\rangle+\left\langle a_{j}(\mathbf{k}) \frac{\partial a_{j^{\prime}}\left(\mathbf{k}^{\prime}\right)}{\partial t}\right\rangle=(22) \\
\varepsilon \int_{\mathrm{R}^{6}} \mathcal{H}_{j m n}^{\mathbf{k p q}}\left\langle a_{m}(\mathbf{p}) a_{n}(\mathbf{q}) a_{j^{\prime}}\left(\mathbf{k}^{\prime}\right)\right\rangle e^{i \Omega_{k, p q} t} \delta_{k, p q} d \mathbf{p} d \mathbf{q} \\
+ \\
\varepsilon \int_{\mathrm{R}^{6}} \mathcal{H}_{j^{\prime} m n}^{\mathbf{k}^{\prime} \mathbf{p q}}\left\langle a_{m}(\mathbf{p}) a_{n}(\mathbf{q}) a_{j}(\mathbf{k})\right\rangle e^{i \Omega_{k^{\prime}, p q} t} \delta_{k^{\prime}, p q} d \mathbf{p} d \mathbf{q} .
\end{gathered}
$$

A hierarchy of equations will clearly appear which gives for the third order moment equation

$$
\begin{aligned}
& \frac{\partial\left\langle a_{j}(\mathbf{k}) a_{j^{\prime}}\left(\mathbf{k}^{\prime}\right) a_{j^{\prime \prime}}\left(\mathbf{k}^{\prime \prime}\right)\right\rangle}{\partial t}= \\
& \varepsilon \int_{\mathrm{R}^{6}} \mathcal{H}_{j m n}^{\mathbf{k p q}}\left\langle a_{m}(\mathbf{p}) a_{n}(\mathbf{q}) a_{j^{\prime}}\left(\mathbf{k}^{\prime}\right) a_{j^{\prime \prime}}\left(\mathbf{k}^{\prime \prime}\right)\right\rangle e^{i \Omega_{k, p q} t} \delta_{k, p q} d \mathbf{p} d \mathbf{q} \\
& + \\
& \varepsilon \int_{\mathbf{R}^{6}} \mathcal{H}_{j^{\prime} m n}^{\mathbf{k}^{\prime} \mathbf{p q}}\left\langle a_{m}(\mathbf{p}) a_{n}(\mathbf{q}) a_{j}(\mathbf{k}) a_{j^{\prime \prime}}\left(\mathbf{k}^{\prime \prime}\right)\right\rangle e^{i \Omega_{k^{\prime}, p q} t} \delta_{k^{\prime}, p q} d \mathbf{p} d \mathbf{q} \\
& + \\
& \varepsilon \int_{\mathrm{R}^{6}} \mathcal{H}_{j^{\prime \prime} m n}^{\mathbf{k}^{\prime \prime} \mathbf{p q}}\left\langle a_{m}(\mathbf{p}) a_{n}(\mathbf{q}) a_{j}(\mathbf{k}) a_{j^{\prime}}\left(\mathbf{k}^{\prime}\right)\right\rangle e^{i \Omega_{k^{\prime \prime}, p q}{ }^{t}} \delta_{k^{\prime \prime}, p q} d \mathbf{p} d \mathbf{q} .
\end{aligned}
$$

At this stage, we may write the fourth order moment in terms of a sum of the fourth order cumulant plus products of second order ones, but a natural closure arises for times asymptotically large (Benney and Saffman, 1966; Benney and Newell, 1967, 1969; Newell et al., 2001). In this case, several terms do not contribute at large times like, in particular, the fourth order cumulant which is not a resonant term. In other words, the nonlinear regeneration of third order moments depends essentially on products of second order moments. The time scale separation imposes a condition of applicability of wave turbulence which has to be checked in fine (see e.g., Nazarenko, 2007). After integration in time, we are left with

$$
\begin{gathered}
\left\langle a_{j}(\mathbf{k}) a_{j^{\prime}}\left(\mathbf{k}^{\prime}\right) a_{j^{\prime \prime}}\left(\mathbf{k}^{\prime \prime}\right)\right\rangle= \\
\varepsilon \int_{\mathrm{R}^{6}} \mathcal{H}_{j m n}^{\mathbf{k p q}}\left(\left\langle a_{m}(\mathbf{p}) a_{n}(\mathbf{q})\right\rangle\left\langle a_{j^{\prime}}\left(\mathbf{k}^{\prime}\right) a_{j^{\prime \prime}}\left(\mathbf{k}^{\prime \prime}\right)\right\rangle\right. \\
+\left\langle a_{m}(\mathbf{p}) a_{j^{\prime}}\left(\mathbf{k}^{\prime}\right)\right\rangle\left\langle a_{n}(\mathbf{q}) a_{j^{\prime \prime}}\left(\mathbf{k}^{\prime \prime}\right)\right\rangle \\
\left.+\left\langle a_{m}(\mathbf{p}) a_{j^{\prime \prime}}\left(\mathbf{k}^{\prime \prime}\right)\right\rangle\left\langle a_{n}(\mathbf{q}) a_{j^{\prime}}\left(\mathbf{k}^{\prime}\right)\right\rangle\right) \Delta\left(\Omega_{k, p q}\right) \delta_{k, p q} d \mathbf{p} d \mathbf{q} \\
+
\end{gathered}
$$




$$
\begin{gathered}
\varepsilon \int_{\mathrm{R}^{6}} \mathcal{H}_{j^{\prime} m n}^{\mathbf{k}^{\prime} \mathbf{p q}}\left(\left\langle a_{m}(\mathbf{p}) a_{n}(\mathbf{q})\right\rangle\left\langle a_{j}(\mathbf{k}) a_{j^{\prime \prime}}\left(\mathbf{k}^{\prime \prime}\right)\right\rangle\right. \\
+\left\langle a_{m}(\mathbf{p}) a_{j}(\mathbf{k})\right\rangle\left\langle a_{n}(\mathbf{q}) a_{j^{\prime \prime}}\left(\mathbf{k}^{\prime \prime}\right)\right\rangle \\
\left.+\left\langle a_{m}(\mathbf{p}) a_{j^{\prime \prime}}\left(\mathbf{k}^{\prime \prime}\right)\right\rangle\left\langle a_{n}(\mathbf{q}) a_{j}(\mathbf{k})\right\rangle\right) \Delta\left(\Omega_{k^{\prime}, p q}\right) \delta_{k^{\prime}, p q} d \mathbf{p} d \mathbf{q} \\
+ \\
\varepsilon \int_{\mathrm{R}^{6}} \mathcal{H}_{j^{\prime \prime} m n}^{\mathbf{k}^{\prime \prime} \mathbf{p q}}\left(\left\langle a_{m}(\mathbf{p}) a_{n}(\mathbf{q})\right\rangle\left\langle a_{j}(\mathbf{k}) a_{j^{\prime}}\left(\mathbf{k}^{\prime}\right)\right\rangle\right. \\
+\left\langle a_{m}(\mathbf{p}) a_{j}(\mathbf{k})\right\rangle\left\langle a_{n}(\mathbf{q}) a_{j^{\prime}}\left(\mathbf{k}^{\prime}\right)\right\rangle \\
\left.+\left\langle a_{m}(\mathbf{p}) a_{j^{\prime}}\left(\mathbf{k}^{\prime}\right)\right\rangle\left\langle a_{n}(\mathbf{q}) a_{j}(\mathbf{k})\right\rangle\right) \Delta\left(\Omega_{k^{\prime \prime}, p q}\right) \delta_{k^{\prime \prime}, p q} d \mathbf{p} d \mathbf{q},
\end{gathered}
$$

where

$\Delta\left(\Omega_{k, p q}\right)=\int_{0}^{t \gg 1 / \omega} e^{i \Omega_{k, p q} t^{\prime}} d t^{\prime}=\frac{e^{i \Omega_{k, p q} t}-1}{i \Omega_{k, p q}}$.

After integration in wave vectors $\mathbf{p}$ and $\mathbf{q}$ and simplification, we get

$$
\begin{aligned}
& \left\langle a_{j}(\mathbf{k}) a_{j^{\prime}}\left(\mathbf{k}^{\prime}\right) a_{j^{\prime \prime}}\left(\mathbf{k}^{\prime \prime}\right)\right\rangle= \\
& \varepsilon \Delta\left(\Omega_{k k^{\prime} k^{\prime \prime}}\right) \delta_{k k^{\prime} k^{\prime \prime}} \\
& \left(\mathcal{H}_{j m n}^{\mathbf{k}-\mathbf{k}^{\prime}-\mathbf{k}^{\prime \prime}} q_{m j^{\prime}}\left(\mathbf{k}^{\prime}\right) q_{n j^{\prime \prime}}\left(\mathbf{k}^{\prime \prime}\right)+\mathcal{H}_{j m n}^{\mathbf{k}-\mathbf{k}^{\prime \prime}-\mathbf{k}^{\prime}} q_{m j^{\prime \prime}}\left(\mathbf{k}^{\prime \prime}\right) q_{n j^{\prime}}\left(\mathbf{k}^{\prime}\right)\right. \\
& +\mathcal{H}_{j^{\prime} m n}^{\mathbf{k}^{\prime}-\mathbf{k}-\mathbf{k}^{\prime \prime}} q_{m j}(\mathbf{k}) q_{n j^{\prime \prime}}\left(\mathbf{k}^{\prime \prime}\right)+\mathcal{H}_{j^{\prime} m n}^{\mathbf{k}^{\prime}-\mathbf{k}^{\prime \prime}-\mathbf{k}} q_{m j^{\prime \prime}}\left(\mathbf{k}^{\prime \prime}\right) q_{n j}(\mathbf{k}) \\
& \left.+\mathcal{H}_{j^{\prime \prime m n}}^{\mathbf{k}^{\prime \prime}-\mathbf{k}-\mathbf{k}^{\prime}} q_{m j}(\mathbf{k}) q_{n j^{\prime}}\left(\mathbf{k}^{\prime}\right)+\mathcal{H}_{j^{\prime \prime} m n}^{\mathbf{k}^{\prime \prime}-\mathbf{k}^{\prime}-\mathbf{k}} q_{m j^{\prime}}\left(\mathbf{k}^{\prime}\right) q_{n j}(\mathbf{k})\right) .
\end{aligned}
$$

The symmetries (19) lead to

$$
\begin{aligned}
& \left\langle a_{j}(\mathbf{k}) a_{j^{\prime}}\left(\mathbf{k}^{\prime}\right) a_{j^{\prime \prime}}\left(\mathbf{k}^{\prime \prime}\right)\right\rangle= \\
& 2 \varepsilon \Delta\left(\Omega_{k k^{\prime} k^{\prime \prime}}\right) \delta_{k k^{\prime} k^{\prime \prime}}\left(\mathcal{H}_{j m n}^{\mathbf{k}-\mathbf{k}^{\prime}-\mathbf{k}^{\prime \prime}} q_{m j^{\prime}}\left(\mathbf{k}^{\prime}\right) q_{n j^{\prime \prime}}\left(\mathbf{k}^{\prime \prime}\right)\right. \\
& \left.+\mathcal{H}_{j^{\prime} m n}^{\mathbf{k}^{\prime}-\mathbf{k}-\mathbf{k}^{\prime \prime}} q_{m j}(\mathbf{k}) q_{n j^{\prime \prime}}\left(\mathbf{k}^{\prime \prime}\right)+\mathcal{H}_{j^{\prime \prime} m n}^{\mathbf{k}^{\prime \prime}-\mathbf{k}-\mathbf{k}^{\prime}} q_{m j}(\mathbf{k}) q_{n j^{\prime}}\left(\mathbf{k}^{\prime}\right)\right) .
\end{aligned}
$$

The latter expression may be introduced into Eq. (22). We take the long time limit for which

$\Delta(x) \rightarrow \pi \delta(x)+i \mathcal{P}(1 / x)$,

with $\mathcal{P}$ the principal value of the integral. Note that this limit, at this level, introduces irreversibility in time. We finally find the asymptotically exact wave kinetic equations

$$
\begin{gathered}
\frac{\partial q_{j j^{\prime}}(\mathbf{k})}{\partial t}=4 \\
\qquad \varepsilon^{2} \int_{\mathrm{R}^{6}} \delta_{k, p q} \delta\left(\Omega_{k, p q}\right) \mathcal{H}_{j m n}^{\mathbf{k p q}} \\
{\left[\mathcal{H}_{m r s}^{\mathbf{p}-\mathbf{q}-\mathbf{k}} q_{r n}(\mathbf{q}) q_{j^{\prime} s}(\mathbf{k})+\mathcal{H}_{n r s}^{\mathbf{q}-\mathbf{p k}} q_{r m}(\mathbf{p}) q_{j^{\prime} s}(\mathbf{k})\right.} \\
\left.+\mathcal{H}_{j^{\prime} r s}^{-\mathbf{k}-\mathbf{p}-\mathbf{q}} q_{r m}(\mathbf{p}) q_{s n}(\mathbf{q})\right] d \mathbf{p} d \mathbf{q} .
\end{gathered}
$$

These general 3-D wave kinetic equations are valid in principle for any situation where three-wave interaction processes are dominant; only the form of $\mathcal{H}$ will be different. Equation for the total energy is found with the trace of the tensor density, $q_{j j}(\mathbf{k})$, whereas other inviscid invariants are obtained by including non diagonal terms.

\subsection{Wave kinetic equations}

Equation (29) is the wave kinetic equation for the spectral tensor components. We see that the mechanism of nonlinear transfer is based on resonance since we need to satisfy the relations

$$
\begin{aligned}
\omega_{k} & =\omega_{p}+\omega_{q}, \\
\mathbf{k} & =\mathbf{p}+\mathbf{q} .
\end{aligned}
$$

The solutions define the resonant manifolds which may have different forms according to the flow. For example in the limit of weakly compressible MHD, when the sound speed is much greater than the Alfvén speed, it is possible to find (for the shape of the resonant manifolds in the 3-D kspace) spheres or tilted planes for Fast-Fast-Alfvén and FastFast-Slow wave interactions, and rays (a degenerescence of the resonant manifolds) for Fast-Fast-Fast wave interactions (Galtier et al., 2001). We also find planes perpendicular to the uniform magnetic field $\mathbf{B}_{\mathbf{0}}$ for Slow-Slow-Slow, Slow-SlowAlfvén, Slow-Alfvén-Alfvén or Alfvén-Alfvén-Alfvén wave interactions; it is a similar situation to incompressible MHD turbulence (since, at first order, slow waves have the same frequency as Alfvén waves) for which the resonant manifolds foliate the Fourier space.

The representation of the resonant manifolds is always interesting since it gives an idea of how the spectral densities can redistribute along the mean magnetic field direction whose main effect is the nonlinear transfer reduction along its direction (Matthaeus et al., 1996). The previous finding was confirmed by a detailed analysis of wave compressible MHD turbulence (Kuznetsov, 2001; Chandran, 2005) in the small $\beta$ limit where the wave kinetic equations were derived as well as their exact power law solutions. The situation for electron and Hall MHD is more subtle and there is no simple picture for the resonant manifolds as the one found for MHD. In this case, it is nevertheless important to check the existence of a solution to the resonance condition to justify the domination of three-wave interaction processes over higher order (four-wave) processes.

The form of the wave kinetic Eq. (29) is the most general for a dispersive problem as in electron MHD where we find whistler waves. The incompressible MHD system constitutes a unique case of pseudo-dispersive waves for which wave turbulence applies. In this particular case, some symmetries are lost and the principal value terms, which cancel each other in general, remain present. For that reason, incompressible MHD may be seen as a singular limit of incompressible Hall MHD (Galtier, 2006b).

\subsection{Finite flux solutions}

The most spectacular characteristic of the wave kinetic equations is their ability to provide exact finite flux solutions. These solutions are found after applying to the wave kinetic equations a conformal transform proposed first by Zakharov 
and Filonenko (1966) for four-wave interaction processes in isotropic turbulence (see also, Zakharov, 1967). These solutions are the so-called Kolmogorov spectra. Anisotropy is almost always found in magnetized plasmas and a bihomogeneous conformal transform is then more appropriate (Kuznetsov, 1972). To simplify the analytical treatment, it is necessary to assume an axially symmetric medium; then the wave kinetic Eq. (29) write

$$
\begin{aligned}
& \frac{\partial \tilde{q}_{j j^{\prime}}\left(k_{\perp}, k_{\|}\right)}{\partial t}=4 \pi \varepsilon^{2} \int_{\mathrm{R}^{6}} \delta_{k, p q} \delta\left(\Omega_{k, p q}\right) \tilde{\mathcal{H}}_{j m n}^{\mathbf{k p q}} \\
& {\left[\tilde{\mathcal{H}}_{m r s}^{\mathbf{p}-\mathbf{q}-\mathbf{k}} \tilde{q}_{r n}\left(q_{\perp}, q_{\|}\right) \tilde{q}_{j^{\prime} s}\left(k_{\perp}, k_{\perp}\right)\right.} \\
&+\tilde{\mathcal{H}}_{n r s}^{\mathbf{q}-\mathbf{p k}} \tilde{q}_{r m}\left(p_{\perp}, p_{\|}\right) \tilde{q}_{j^{\prime} s}\left(k_{\perp}, k_{\|}\right) \\
&\left.+\tilde{\mathcal{H}}_{j^{\prime} r s}^{-\mathbf{k}-\mathbf{p}-\mathbf{q}} \tilde{q}_{r m}\left(p_{\perp}, p_{\|}\right) \tilde{q}_{s n}\left(q_{\perp}, q_{\|}\right)\right] d p_{\perp} d p_{\|} d q_{\perp} d q_{\|},
\end{aligned}
$$

where $\tilde{q}_{j j^{\prime}}\left(k_{\perp}, k_{\|}\right)=q_{j j^{\prime}}(\mathbf{k}) /\left(2 \pi k_{\perp}\right)$ and $\tilde{\mathcal{H}}$ is a geometric operator. Note that we have performed an integration over the polar angle. Except for incompressible MHD for which the wave kinetic equations simplify because of the total absence of nonlinear transfer along the parallel direction (the direction along $\mathbf{B}_{\mathbf{0}}$ ), in general we have to deal with the perpendicular and parallel directions. In this case, a further assumption may be made if we recall that magnetized plasmas are strongly anisotropic under the influence of a strong external magnetic field $B_{0}$. In this case, we may write the wave kinetic equations in the limit $k_{\perp} \gg k_{\|}$. The system of integrodifferential equations obtained is then sufficiently reduced to provide the exact power law solutions. We apply the conformal transformation to the equations for inviscid invariants (total energy, magnetic helicity...)

$$
\begin{aligned}
& p_{\perp} \rightarrow k_{\perp}^{2} / p_{\perp}, \\
& q_{\perp} \rightarrow k_{\perp} q_{\perp} / p_{\perp}, \\
& p_{\|} \rightarrow k_{\|}^{2} / p_{\|}, \\
& q_{\|} \rightarrow k_{\|} q_{\|} / p_{\|},
\end{aligned}
$$

and we search for stationary solutions in the power law form $k_{\perp}^{-n} k_{\|}^{-m}$. Basically two types of solutions are found: the fluxless solution, also called the thermodynamic equilibrium solution, which corresponds to the equipartition state for which the flux is zero, and the finite flux solution which is the most interesting one. During the last decades many papers have been devoted to the finding of these finite flux solutions for isotropic as well as anisotropic wave turbulence (Zakharov et al., 1992). Recently, and thanks to the high numerical resolution now available, a new challenge has appeared in wave turbulence. Indeed, the study of incompressible MHD wave turbulence (Galtier et al., 2000) suggested that the development of the finite flux solution of the wave kinetic equations may be preceded by a front characterized by a spectrum with a significantly steeper scaling law. This finding is in contradiction with the theory proposed by Falkovich and Shafarenko (1991) on the nonlinear front propagation. The same observation was also made for the inverse cascade in the nonlinear Schrodinger equation (Lacaze et al., 2001) and a detailed analysis was given by Connaughton et al. (2003). In this paper, the anomalous spectrum in the wake was investigated in the limit of strongly local interactions where the usual wave kinetic equations can be approximated by a PDE. Some answers have been given but their generalization to wave turbulence is still an open problem.

\section{Results and predictions from wave turbulence theo- ries}

\subsection{Incompressible MHD}

We start to summarize the results of wave turbulence in the incompressible MHD case for which the wave kinetic equations are singular in the sense that the principal value terms remain except for the Elsässer energies $E^{ \pm}$. As it was explained, the origin of this particularity is the pseudodispersive nature of Alfvén waves which are a unique case where wave turbulence theory applies. In the limit of strongly anisotropic turbulence for which $k_{\perp} \gg k_{\|}$, finite flux solutions were found for the stationary energy spectra of Eq. (32) with the following wavenumber dependence (Galtier et al., 2000)

$E^{ \pm}\left(k_{\perp}, k_{\|}\right) \sim f_{ \pm}\left(k_{\|}\right) k_{\perp}^{n_{ \pm}}$

with

$n_{+}+n_{-}=-4$

$f_{ \pm}$are arbitrary functions of $k_{\|}$and the power law indices satisfy the condition of locality

$-3<n_{ \pm}<-1$.

This solution are fundamentally linked to the resonance condition which implies the total absence of nonlinear transfer along the $\mathbf{B}_{\mathbf{0}}$ direction. The solutions $E^{ \pm}$have to be understood as the energy spectra associated to the Elsässer fields. In other words, the general case of unbalanced turbulence is considered here.

The locality of interactions is an important issue in plasma turbulence. According to some recent works in isotropic turbulence, nonlinear interactions seem to be more non local in MHD than in a pure hydrodynamics in the sense that the transfer of energy from the velocity field to the magnetic field may be a highly non local process in Fourier space (Alexakis et al., 2005). The situation is different when we deal with anisotropic turbulence: in this case interactions (between perpendicular wavevectors) are mainly local (Alexakis et al., 2007). In wave turbulence, the condition Eq. (36) has to be satisfied to validate the exact power law solutions and avoid any divergence due to nonlocal contributions. In practice, numerical simulations of the wave kinetic equations 
have clearly shown that the solutions corresponding to local interactions are attractive (Galtier et al., 2000).

Recently, it was realized that the wave kinetic equations found in the anisotropic limit may be recovered without the wavenumber condition $k_{\perp} \gg k_{\|}$if initially we retain the shear-Alfvén waves only (Galtier and Chandran, 2006). Therefore, the finite flux solution may be extended to the entire wavenumber space which renders its detection easier. This idea was tested recently against numerical simulations (Perez and Boldyrev, 2008) and a spectral signature of wave turbulence was apparently found.

\subsection{Compressible MHD}

We turn now to the compressible case for which two limits have been analyzed. The first case (case I) is the one for which the plasma (thermal) pressure is assumed to be small as compared to the magnetic pressure (small $\beta$ limit) and where three-wave interaction processes that do not involve slow waves are neglected (Kuznetsov, 2001). In the second case (case II) for which we still have $\beta \ll 1$, slow waves are neglected and a constant density is imposed (Chandran, 2005). In both situations the general finite flux solutions are not obvious to express.

In case I, when only interactions between Alfvén and slow waves are kept, a wave energy spectrum in $\sim k_{\perp}^{-2} k_{\|}^{-5 / 2}$ is found which corresponds to a (finite) constant energy flux solution. It is claimed that the addition of the interactions with the fast waves will lead to the same solution since the dynamics tends to produce strongly anisotropic distributions of the waves concentrated in $\mathrm{k}$-space within a narrow-angle cone in the $\mathbf{B}_{\mathbf{0}}$ direction $\left(k_{\perp} \ll k_{\|}\right)$. Under this conditions, the fast waves coincide with the Alfvén waves.

In case II, the general wave kinetic equations do not allow us to find exact power law solutions. However, when only Fast-Fast-Fast interactions are kept it is possible to find a finite flux solution for the fast wave 1-D energy spectrum which scales as $\sim f(\theta) k^{-3 / 2}$, where $f(\theta)$ is an arbitrary function of the angle $\theta$ between the wave vector $\mathbf{k}$ and the uniform magnetic field $\mathbf{B}_{\mathbf{0}}$. Numerical simulations of the general wave kinetic equations are made to find a behavior according, for example, to the angle $\theta$. A solution close to $k_{\perp}^{-2}$ is found for the Alfvén wave 2-D energy spectrum (for different fixed $k_{\|}$) when $k_{\perp} \gg k_{\|}$. The $k$-spectra plotted at $\theta=45^{\circ}$ reveal a fast wave 1-D energy spectrum in $\sim k^{-3 / 2}$ and an steeper Alfvén wave spectrum, while for a small angle $\left(\theta=7.1^{\circ}\right)$ both spectra follow the same scaling law steeper than $k^{-3 / 2}$ (Chandran, 2005).

\subsection{Electron MHD}

The electron MHD equations under the influence of a strong uniform magnetic field $B_{0}$ exhibit dispersive whistler waves. The wave turbulence regime was analyzed in the incompressible case (Galtier and Bhattacharjee, 2003, 2005) and the wave kinetic equations were found by using a complex helicity decomposition. The strong anisotropic $\left(k_{\perp} \gg k_{\|}\right)$finite flux solutions correspond to

$E\left(k_{\perp}, k_{\|}\right) \sim k_{\perp}^{-5 / 2} k_{\|}^{-1 / 2}$

for the 2-D magnetic energy spectrum, and

$H\left(k_{\perp}, k_{\|}\right) \sim k_{\perp}^{-7 / 2} k_{\|}^{-1 / 2}$

for the 2-D magnetic helicity spectrum. As for the other cases presented above, a direct cascade was found for the energy. In particular, it was shown that contrary to MHD the wave kinetic equations, which involve three-wave interaction processes, are characterized by a nonlinear transfer that decreases linearly with $k_{\|}$; for $k_{\|}=0$, the transfer is found to be exactly null. Thus the 2-D modes (also called sometimes slow modes) decouple from the three-dimensional whistler waves. Such decoupling is found in a variety of problems as in rotating turbulence (Galtier, 2003; Sagaut and Cambon, 2008).

\subsection{Hall MHD}

The last example exposed in this paper is the Hall MHD case which incorporates both the standard MHD and the electron MHD approximations. This system is much heavier to analyze in the regime of wave turbulence and it is only recently that a theory has been proposed (Galtier, 2006b). The general theory emphasizes the fact that the large scale limit of standard MHD becomes singular with the apparition of new type of terms, the principal value terms. Of course, the large scale and small scale limits tend to the appropriate theories developed previously, but in addition it is possible to describe the connection between them at intermediate scales (scales of the order of the ion skin depth $d_{i}$ ). For example, moderate anisotropy is predicted at this intermediate scales whereas it is much stronger for other scales. It is also interesting to note that the small scale limit gives a system of equations richer than the pure electron MHD system (Galtier and Bhattacharjee, 2003) with the possibility to describe the sub-dominant kinetic energy dynamics. Exact power law solution for the kinetic energy spectrum is also found for ion cyclotron wave turbulence which scales as

$E\left(k_{\perp}, k_{\|}\right) \sim k_{\perp}^{-5 / 2} k_{\|}^{-1 / 2}$.

To date, the wave kinetic equations of Hall MHD have not been simulated numerically even in its simplified form (when helicity terms are discarded). It is an essential step to understand much better the dynamics at intermediate scales.

\section{Conclusions}

\subsection{Summary}

Waves and turbulence are two fundamental ingredients of many magnetized plasmas. The most spectacular illustration 
of such characteristics is probably given by the latest observations of the Sun's atmosphere with the orbital solar observatory Hinode launched at the end of 2006. For the first time, detection of Alfvén waves is made through the small oscillations of many thin structures called threads. In the meantime the highly dynamical nature of coronal loops is revealed by non-thermal velocities detected with spectrometers. These findings are considered as a remarkable step in our understanding of the solar coronal heating; for that reason, new models including waves and turbulence may be a promising way to heat the solar corona in addition to other models based on physical processes like magnetic reconnection (Regnier et al., 2008).

The interplanetary medium is another example of magnetized plasma where waves and turbulence are detected. In this framework, the origin of the so-called "dissipative range", i.e. the extension of the turbulent inertial range beyond a fraction of hertz, is currently one of the main issue discussed in the community. Although a final answer is not given yet, wave turbulence is a promising regime to understand the inner solar wind dynamics in the sense that it gives exact results in regards to the possible multiscale behavior of magnetized plasmas as well as the intensity of the anisotropic transfer between modes.

The main feature of magnetized plasmas in the regime of wave turbulence is the omnipresence of anisotropy and the possibility to have different spectral scaling laws according to the space direction. To achieve a proper comparison between observational data and theoretical predictions, not only in situ measurements are necessary, but multipoints data have also to be accessible. It is at this price that the true nature of magnetized plasmas will be revealed. The magnetosphere is the first medium where it is possible to perform such a comparison thanks to Cluster (Sahraoui et al., 2006).

\subsection{Coexistence of wave and strong turbulence}

Numerical simulation is currently the main tool to improve our knowledge on wave turbulence in magnetized plasmas since we are still limited by the observational (single point) data. Two types of simulations are available: the simulation of the wave kinetic equations and the so-called direct numerical simulation of the original MHD-type equations. In the former case, it is a way to find for instance the spectral scaling laws when the wave kinetic equations are too complicated to get the exact solutions after application of the usual conformal transform. An example is given by compressible MHD: in this case, the numerics revealed a change of power law in the energy spectrum with the angle between the wave vector and the mean magnetic field $\mathbf{B}_{\mathbf{0}}$ (Chandran, 2005). These numerical simulations may also be useful to investigate wave turbulence when an external forcing is applied like in incompressible MHD (Galtier and Nazarenko, 2008).
For direct numerical simulation, the challenge is slightly different: indeed, in this case the main goal is the measure of the transition between strong and wave turbulence, and thus between isotropic and anisotropic turbulence. The former regime has been extensively studied since more than three decades whereas the latter is still a young topics. The main topic of such a simulation is also to find general properties that could help us to understand the single point measurements made in natural plasmas. We arrive here at the heart of current issues in wave turbulence. One of the most important points emphasized by recent direct numerical simulations in incompressible MHD is the coexistence of wave and strong turbulence (Bigot et al., 2008b). This characteristic should not be a surprise since basically wave turbulence is a perturbative theory which must satisfy condition of applicability. In this case, the 2-D state $\left(k_{\|}=0\right)$ evolves differently from the 3-D modes $\left(k_{\|}>0\right)$ since the former case is characterized by strong turbulence and the latter mainly by wave turbulence. This comment, although simple, is fundamental and not really taken into account by the community. Indeed, wave turbulence will not be revealed in natural as well as simulated plasmas as long as strong and wave turbulence are not separated. A recent work (Bigot et al., 2008b) shows that the true nature of wave turbulence in incompressible MHD is revealed when the 2-D state is filtered out. For example, much steeper energy spectra are found for 3-D modes compared to the 2-D state. The $k_{\perp}^{-2}$ scaling is still a challenging solution for direct numerical simulations since it requires a wide inertial range to satisfy the condition $k_{\perp} \gg k_{\|}$, but the latest results are promising since they are already very close to it. Note that these spectra are only visible at a fixed $k_{\|}$ and it is hidden in a $k_{\perp}^{-5 / 3}$ if it is plotted after summation over the $k_{\|}$. The same simulations also show (as expected) an equipartition between kinetic and magnetic energies for 3D modes whereas the 2-D state exhibits a state dominated by the magnetic energy (Alfvén ratio smaller than one, i.e. a ratio between kinetic and magnetic energies smaller than one). Information about structures and the filamentation of current and vorticity sheets are also reported.

In the light of such simulations, in particular the direct one, new questions arise for natural plasmas. For the solar wind, does the $-5 / 3$ spectrum correspond to a bias since single point measurements automatically mean a 1-D spectrum? Is the small Alfvén ratio, around 0.5 at one $\mathrm{AU}$ (Bruno and Carbone, 2005), due essentially to the 2-D state? Is intermittency mainly due to the $2 \mathrm{D}$ state or interactions between the 2-D state and the 3-D modes? Only multispacecraft mission - like Cluster - can answer such questions. The apparent condensation into filaments discussed above reminds ones of the coronal loops observed on the Sun. Could the coronal strands be the end result of a nonlinear process occurring in anisotropic MHD turbulence? Current and future missions will certainly help us to answer these questions. 


\subsection{Theoretical issues in anisotropic turbulence}

Anisotropic turbulence is characterized by the possibility to have different spectral scaling laws according to the space direction. Progress has been made during the last decade to quantify heuristically such anisotropy. It is mainly based on the assumption of a (critical) balance between the wave time and the eddy-turnover time. Principal applications are MHD and electron MHD, in the incompressible case, in which we find respectively, $k_{\|} \sim k_{\perp}^{2 / 3}$ and $k_{\|} \sim k_{\perp}^{1 / 3}$ (see e.g., Goldreich and Sridhar, 1995). Although these scaling laws are reported by several direct numerical simulations, the associated energy spectrum is still a subject of discussion in regards to the $1-\mathrm{D}$ versus 2-D scaling (as explained above). This subject of research is probably one of the most important for the future.

It is fundamental to remind at this point that the scalings proposed between parallel and perpendicular wavenumbers are by nature heuristic and we should not invoke the word "theory" as often reported (and reserve it, for example, to wave turbulence). Contrary to hydrodynamics where the $-5 / 3$-spectrum is supported indirectly by a theory - the so called four-fifth's law - and where the world "theory" is in this sense more justified, to date in anisotropic MHD there is no equivalent, nor in anisotropic electron/Hall MHD, in the incompressible as well as the compressible case. Note, however, the recent progress in incompressible electron and Hall MHD where the equivalent of the four-fifth's law is now derived (Galtier, 2008a,b) and put therefore the $-7 / 3$ energy spectrum law of strong turbulence (Biskamp et al., 1996) on a more solid foundation (since it is compatible dimensionally). The generalization of the isotropic four-third's law to anisotropy MHD turbulence may be the next fundamental step to reach in order to give, for the first time, a theory of strong anisotropic turbulence.

Acknowledgements. This work was supported by the ANR project no. 06-BLAN-0363-01 "HiSpeedPIV" and by the INSU/PNST Program.

Edited by: T. Passot

Reviewed by: G. Belmont

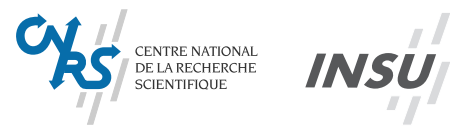

The publication of this article is financed by CNRS-INSU.

\section{References}

Abdurakhimov, L. V., Brazhnikov, M. Y., and Levchenko, A. A.: Statistic of capillary waves on the surface of liquid hydrogen in a turbulent regime, J. Low Temp. Phys., 150, 431-434, 2008.

Alexakis, A., Bigot, B., Politano, H., and Galtier, S.: Anisotropic fluxes and nonlocal interactions in magne- tohydrodynamic turbulence, Phys. Rev. E., 76, 056313, doi:10.1103/PhysRevE.76.056313, 2007.

Alexakis, A., Mininni, P. D., and Pouquet, A.: Shell-to-shell energy transfer in magnetohydrodynamics. I. Steady state turbulence, Phys. Rev. E., 72, 046301, 2005.

Alexandrova, O., Carbone, V., Veltri, P., and Sorriso-Valvo, L.: Small-scale energy cascade of the solar wind turbulence, Astrophys. J., 674, 1153-1157, 2008.

Armstrong, J. W., Coles, W. A., Rickett, B. J., and Kojima, M.: Observations of field-aligned density fluctuations in the inner solar wind, Astrophys. J., 358, 685-692, 1990.

Aschwanden, M.J., Poland, A.I., and Rabin, D.M.: The new solar corona, Annu. Rev. Astron. Astrophys., 39, 175-210, 2001.

Bale, S. D., Kellogg, P. J., Mozer, F. S., Horbury, T. S., and Reme, H.: Measurement of the electric field fluctuation spectrum of MHD turbulence, Phys. Rev. Lett., 94, 215002, doi:10.1103/PhysRevLett.94.215002, 2005.

Barnes, A.: Interplanetary Alfvénic fluctuations: a stochastic model, J. Geophys. Res., 86, 7498-7506, 1981.

Batchelor, G. K.: The theory of homogeneous turbulence, Cambridge University Press, 1953

Belcher, J. W. and Davis, L.: Large-Amplitude Alfvén waves in the interplanetary medium, J. Geophys. Res., 76, 3534-3563, 1971.

Belmont, G. and Rezeau, L.: Magnetopause reconnection induced by magnetosheath Hall-MHD fluctuations, J. Geophys. Res., 106, 10751-10760, 2001.

Benney, J., and Saffman, P. G.: Nonlinear interactions of random waves in a dispersive medium, Proc. Roy. Soc. A., 289, 301-320, 1966.

Benney, J. and Newell, A. C.: Sequential time closures for interacting random wavers, J. Maths. Phys., 46, 363-393, 1967.

Benney, J. and Newell, A. C.: Random wave closures, Studies in Applied Math., 48, 29-53, 1966.

Bhattacharjee, A.: Impulsive magnetic reconnection in the earth's magnetotail and the solar corona, Ann. Rev. Astron. Astrophys., 42, 365-384, 2004.

Bieber, J. W., Wanner, W., and Matthaeus, W. H.: Dominant twodimensional solar wind turbulence with implications for cosmic ray transport, J. Geophys. Res., 101, 2511-2522, 1996.

Bigot, B., Galtier, S., and Politano, H.: Energy decay laws in strongly anisotropic MHD turbulence, Phys. Rev. Lett., 100, 074502, doi:10.1103/PhysRevLett.100.074502, 2008a.

Bigot, B., Galtier, S., and Politano, H.: Development of Anisotropy in Incompressible Magnetohydrodynamic Turbulence, Phys Rev. E., 78, 066301, doi:10.1103/PhysRevE.78.066301, 2008 b.

Bigot, B., Galtier, S., and Politano, H.: An anisotropic turbulent model for solar coronal heating, Astron. Astrophys., 490, 325337, 2008c.

Biskamp, D.: Collision and collisionless magnetic reconnection, Phys. Plasmas, 4, 1964-1968, 1997.

Biskamp, D., Schwarz, E., and Drake, J.F .: 2-D electron magnetohydrodynamic turbulence, Phys. Rev. Lett., 76, 1264-1267, 1996.

Biskamp, D., Schwarz, E., Zeiler, A., Celani, A., and Drake, J. F.: Electron magnetohydrodynamic turbulence, Phys. Plasmas, 6, 751-758, 1999.

Boldyrev, S.: Spectrum of magnetohydrodynamic turbulence, Phys. Rev. Lett., 96, 115002, doi:10.1103/PhysRevLett.96.115002, 2006. 
Brekke, P., Kjeldseth-Moe, O., and Harrison, R. A.: High-velocity flows in an active region loop system observed with the coronal diagnostic spectrometer on SOHO, Sol. Phys., 175, 511-521, 1997.

Bruno, R. and Carbone, V.: The solar wind as a turbulence laboratory, Living Rev. Solar Phys., 2, 1-186, 2005.

Buchlin, É. and Velli, M.: Shell models of RMHD turbulence and the heating of solar coronal loops, Astrophys. J., 662, 701-714, 2007.

Chae, J., Schühle, U., and Lemaire, P.: Sumer measurements of nonthermal motions: constraints on coronal heating mechanism, Astrophys. J., 505, 957-973, 1998.

Chandran, B. D. G.: Weak compressible magnetohydrodynamic turbulence in the solar corona, Phys. Rev. Lett., 95, 265004, doi:10.1103/PhysRevLett.95.265004, 2005.

Chandran, B. D. G.: Strong anisotropic MHD turbulence with cross helicity, Astrophys. J., 685, 646-658, 2008.

Chandrasekhar, S.: The invariant theory of isotropic turbulence in magneto-hydrodynamics, Proc. Roy. Soc. A., 204, 435-449, 1951.

Cho, J. and Vishniac, E. T.: The anisotropy of magnetohydrodynamic Alfvénic turbulence, Astrophys. J., 539, 273-282, 2000.

Cho, J. and Vishniac, E. T.: The anisotropy of electron magnetohydrodynamic turbulence, Astrophys. J., 615, L41-L44, 2004.

Coleman, P. J.: Turbulence, viscosity, and dissipation in the solarwind plasma, Astrophys. J., 153, 371-388, 1968.

Connaughton, C., Newell, A. C., and Pomeau, Y.: Non-stationary spectra of local wave turbulence, Physica D., 184, 64-85, 2003.

Coroniti, F. V., Kennel, C. F., and Scarf, F. L.: Whistler mode turbulence in the disturbed solar wind, J. Geophys. Res., 87, 60296044, 1981.

Cranmer, S. R., van Ballegooijen, A. A., and Edgar, R. J.: Selfconsistent coronal heating and solar wind acceleration from anisotropic magnetohydrodynamic turbulence, Astrophys. J., 171, 520-551, 2007.

Dastgeer, S., Das, A., Kaw, P., and Diamond, P. H.: Whistlerisation and anisotropy in 2-D electron magnetohydrodynamic turbulence, Phys. Plasmas, 7, 571-579, 2000.

David, C., Gabriel, A. H., Bely-Dubau, F., Fludra, A., Lemaire, P., and Wilhelm, K.: Measurement of the electron temperature gradient in a solar coronal hole, Astron. Astrophys., 336, L90L94, 1998.

Denskat, K. U., Beinroth, H. J., and Neubauer, F. M.: Interplanetary magnetic field power spectra with frequencies from $2.410^{-5} \mathrm{~Hz}$ to $470 \mathrm{~Hz}$ from HELIOS-observations during solar minimum conditions., J. Geophys., 54, 60-67, 1983.

Doschek, G. A., Mariska, J. T., Warren, H. P., Brown, C. M., Culhane, J. L., Hara, H., Waranabe, T., Toung, P. R., and Mason, H. E.: Nonthermal velocities in solar active regions observed with the extreme-ultraviolet imaging spectrometer on Hinode, Astrophys. J., 667, L109-L112, 2007.

Dyachenko, S., Newell, A. C., Pushkarev, A. N., and Zakharov, V. E.: Optical turbulence: weak turbulence, condensates and collapsing filaments in the nonlinear Schrödinger equation, Physica D., 57, 96-160, 1992.

Elmegreen, B. G. and Scalo, J.: Interstellar turbulence I: observations and processes, Annu. Rev. Astron. Astrophys., 42, 211273, 2004.

Falcon, É., Laroche, C., and Fauve, S.: Observation of gravity- capillary wave turbulence, Phys. Rev. Lett., 98, 094503, doi:10.1103/PhysRevLett.98.094503, 2007.

Falkovich, G. E. and Shafarenko, A. V.: Nonstationary wave turbulence, J. Nonlinear Sci., 1, 457-480, 1991.

Forsyth, R. J., Balogh, A., Horbury, T. S., Erdös, G., Smith, E. J., and Burton, M. E.: The heliospheric magnetic field at solar minimum: Ulysses observations from pole to pole, Astron. Astrophys., 316, 287-295, 1996.

Frisch, U.: Turbulence, Cambridge University Press, 1995

Galtier, S., Nazarenko, S. V., Newell, A. C., and Pouquet, A. A weak turbulence theory for incompressible MHD, J. Plasma Phys., 63, 447-488, 2000.

Galtier, S., Nazarenko, S. V., and Newell, A. C.: On wave turbulence in MHD, Nonlin. Processes Geophys., 8, 141-150, 2001, http://www.nonlin-processes-geophys.net/8/141/2001/.

Galtier, S., Nazarenko, S. V., Newell, A. C., and Pouquet, A.: Anisotropic turbulence of shear-Alfvén waves, Astrophys. J., 564, L49-L52, 2002.

Galtier, S.: A weak inertial wave turbulence theory, Phys. Rev. E., 68, 015301, doi:10.1103/PhysRevE.68.015301, 2003.

Galtier, S. and Bhattacharjee, A.: Anisotropic weak whistler wave turbulence in electron magnetohydrodynamics, Phys. Plasmas, 10, 3065-3076, 2003

Galtier, S. and Bhattacharjee, A.: Anisotropic wave turbulence in electron MHD, Plasma Phys. Control. Fusion, 47, B691-B701, 2005.

Galtier, S., Pouquet, A., and Mangeney, A.: On the spectral scaling laws for incompressible anisotropic MHD turbulence, Phys. Plasmas, 12, 092310, doi:10.1063/1.2052507, 2005.

Galtier, S.: Multi-scale turbulence in the solar wind, J. Low Temp. Phys., 145, 59-74, 2006a.

Galtier, S.: Wave turbulence in incompressible magnetohydrodynamics, J. Plasma Phys., 72, 721-769, 2006b.

Galtier, S. and Chandran, B. D. G.: Extended spectral scaling laws for shear-Alfvén wave turbulence, Phys. Plasmas, 13, 114505, doi:10.1063/1.2399466, 2006.

Galtier, S. and Buchlin, É.: Multiscale Hall magnetohydrodynamic turbulence in the solar wind, Astrophys. J., 656, 560-566, 2007.

Galtier, S.: von Karman-Howarth equations for Hall Magnetohydrodynamic flows, Phys. Rev. E., 77, 015302(R)-1-4, 2008a.

Galtier, S.: Exact scaling laws for 3D electron MHD turbulence, J. Geophys. Res., 113, A01102, doi:10.1029/2007JA012821, $2008 b$.

Galtier, S. and Nazarenko, S. V.: Large-scale magnetic field sustainment by forced MHD wave turbulence, J. Turbulence, 9(40), 1-10, 2008.

Ghosh, S., Siregar, E., Roberts, D. A., and Goldstein, M. L.: Simulation of high-frequency solar wind power spectra using Hall magnetohydrodynamic, J. Geophys. Res., 101, 2493-2504, 1996.

Goldreich, P. and Reisenegger, A.: Magnetic field decay in isolated neutron stars, Astrophys. J., 395, 250-258, 1992.

Goldreich, P. and Sridhar, S.: Toward a theory of interstellar turbulence. II. Strong alfvénic turbulence, Astrophys. J., 438, 763775,1995

Goldstein, M. L., Roberts, D. A., and Fitch, C. A.: Properties of the fluctuating magnetic helicity in the inertial and dissipative ranges of solar wind turbulence, J. Geophys. Res., 99, 11519-11538, 1994. 
Goldstein, M. L. and Roberts, D. A.: Magnetohydrodynamics turbulence in the solar wind, Phys. Plasmas, 6, 4154-4160, 1999.

Hendrix, D. L. and Van Hoven, G.: Magnetohydrodynamic turbulence and implications for solar coronal heating, Astrophys. J., 467, 887-893, 1996.

Higdon, J. C.: Density fluctuations in the interstellar medium: evidence for anisotropic magnetogasdynamic turbulence. I Model and astrophysical sites, Astrophys. J., 285, 109-123, 1984.

Hollweg, J.V., and Isenberg, P.A.: Generation of the fast solar wind: a review with emphasis on the resonant cyclotron interaction, $\mathrm{J}$. Geophys. Res., 107, 1147-1-37, 2002.

Howes, G. G., Cowley, S. C., Dordland, W., Hammett, G. W., Quataert, E., and Schekochihin, A. A.: A model of turbulence in magnetized plasmas: implications for the dissipation range in the solar wind, J. Geophys. Res., 113, A05103, doi:10.1029/2007JA012665, 2008.

Iroshnikov, P. S.: Turbulence of a conducting fluid in a strong magnetic field, Soviet Astron., 7, 566-571, 1964.

Kingsep, A. S., Chukbar, K. V., and Yankov, V. V.: Electron magnetohydrodynamics, in: Reviews of Plasma Physics, 16, 243-291, Consultant Bureau, New York, 1990.

Klein, L., Bruno, R, Bavassano, B., and Rosenbauer, H: Anisotropy and minimum variance of magnetohydrodynamic fluctuations in the inner heliosphere, J. Geophys. Res., 98, 17461-17466, 1993.

Kolmakov, G. V. and Pokrovsky, V. L.: Stability of weak turbulence spectra in superfluid helium, Physica D., 86, 456-469, 1995.

Kolmakov, G. V., Levchenko, A. A., Brahnikov, M. Y., MezhovDeglin, L. P., Silchenko, A. N., and McClintock, P. V. E.: Quasiadiabatic decay of capillary turbulence on the charged surface of liquid hydrogen, Phys. Rev. Lett., 93, 074501, doi:10.1103/PhysRevLett.93.074501, 2004.

Kolmogorov, A. N.: The local structure of turbulence in incompressible viscous fluid for very high Reynolds numbers, Dokl. Akad. Nauk. SSS, 30, 301-305, 1941.

Kraichnan, R. H.: Inertial range spectrum in hydromagnetic turbulence, Phys. Fluids, 8, 1385-1387, 1965.

Kraichnan, R. H.: Inertial ranges in two-dimensional turbulence, Phys. Fluids, 10, 1417-1423, 1967.

Krishan, V. and Mahajan, S. M.: Magnetic fluctuations and Hall MHD turbulence in the solar wind, J. Geophys. Res., 109, A111051, doi:10.1029/2004JA010496, 2004.

Krommes, J. A.: Fundamental statistical descriptions of plasma turbulence in magnetic fields, Phys. Reports, 360, 1-352, 2002.

Kuznetsov, E. A.: Turbulence of ion sound in a plasma located in a magnetic field, Sov. Phys. J. Exp. Theor. Phys., 35, 310-314, 1972.

Kuznetsov, E. A.: Weak magnetohydrodynamic turbulence of a magnetized plasma, Sov. Phys. J. Exp. Theor. Phys., 93, 10521064, 2001.

Lacaze, R., Lallemand, P., Pomeau, Y., and Rica, S.: Dynamical formation of a Bose-Einstein condensate, Physica D., 152-153, 779-786, 2001.

Laveder, D., Passot, T., and Sulem, P.-L.: Transverse dynamics of dispersive Alfvén waves: I. direct numerical evidence of filamentation, Phys. Plasmas, 9, 293-304, 2002.

Leamon, R. J., Smith, C. W., Ness, N. F., and Matthaeus, W. H.: Observational constraints on the dynamics of the interplanetary magnetic field dissipation range, J. Geophys. Res., 103, 47754787, 1998.
Lithwick, Y. and Goldreich, P.: Imbalanced weak magnetohydrodynamic turbulence, Astrophys. J., 582, 1220-1240, 2003.

Lvov, Y., Nazarenko, S.V, and West, R.: Wave turbulence in BoseEinstein condensates, Physica D., 184, 333-351, 2003.

MacBride, B. T., Smith, C. W., and Forman, M.: The turbulent cascade at $1 \mathrm{AU}$ : energy transfer and the third-order scaling for MHD, Astrophys. J., 679, 1644-1660, 2008.

Markovskii, S. A., Vasquez, B. J., and Smith, C. W.: Statistical analysis of the high-frequency spectral break of the solar wind turbulence at 1 AU, Astrophys. J., 675, 1576-1583, 2008.

Maron, J. and Goldreich, P.: Simulations of incompressible magnetohydrodynamic turbulence, Astrophys. J., 554, 1175-1196, 2001.

Matthaeus, W. H., Ghosh, S., Oughton, S., and Roberts, D. A.: Anisotropic three-dimensional MHD turbulence, J. Geophys. Res., 101, 7619-7629, 1996.

Mininni, P. D., Gómez, D. O., and Mahajan, S. M.: Dynamo action in magnetohydrodynamics and Hall-magnetohydrodynamics, Astrophys. J., 587, 472-481, 2003

Mininni, P. D., Alexakis, A., and Pouquet, A.: Energy transfer in Hall-MHD turbulence: cascades, backscatter, and dynamo action, J. Plasma Physics, 73, 377-401, 2007

Montgomery, D. and Turner, L.: Anisotropic magnetohydrodynamic turbulence in a strong external magnetic field, Phys. Fluids, 24, 825-831, 1981.

Montgomery, D. C. and Matthaeus, W. H.: Anisotropic modal energy transfer in interstellar turbulence, Astrophys. J., 447, 706707, 1995.

Morize, C., Moisy, F., and Rabaud, M.: Decaying grid-generated turbulence in a rotating frame, Phys. Fluids, 17, 095105, 2005.

Nazarenko, S. V., Newell, A. C., and Galtier, S.: Non-local MHD turbulence, Physica D., 152-153, 646-652, 2001.

Nazarenko, S. V.: 2D enslaving of MHD turbulence, New J. Phys., 9, 1-14, 2007.

Newell, A. C., Nazarenko, S. V., and Biven, L.: Wave turbulence and intermittency, Physica D., 152-153, 520-550, 2001.

$\mathrm{Ng}, \mathrm{C}$. S. and Bhattacharjee, A.: Interaction of shear-Alfvén wave packets: implication for weak magnetohydrodynamic turbulence in astrophysical plasmas, Astrophys. J., 465, 845-854, 1996.

Ng, C. S., Bhattacharjee, A., Germaschewski, K., and Galtier, S.: Anisotropic fluid turbulence in the interstellar medium and solar wind, Phys. Plasmas, 10, 1954-1962, 2003.

Nishizuka, N., Shimizu, M., Nakamura, T., Otsuji, K., Okamoto, T. J., Katsukawa, Y., and Shibata, K.: Giant chromospheric anemone jet observed with Hinode and comparison with MHD simulations: evidence of propagating Alfvén waves and magnetic reconnection, Astrophys. J., 683, L83-L86, 2008.

Perez, J.-C. and Boldyrev, S.: On weak and strong magnetohydrodynamic turbulence, Astrophys. J., 672, L61-L64, 2008.

Politano, H. and Pouquet, A.: von-Kárman-Howarth equation for MHD and its consequences on third-order longitudinal structure and correlation functions, Phys. Rev. E., 57, R21-R24, 1998.

Pouquet, A.: Magnetohydrodynamic turbulence, in: Astrophysical fluid dynamics, edited by: Zahn, J.-P. and Zinn-Justin, J., Elsevier science publishers, 139-227, 1993.

Priest, E. R.: Solar magnetohydrodynamics, D. Reidel Pub. Comp., 1982.

Rappazzo, A.F., Velli, M., Einaudi, G., and Dahlburg, R.B.: Coronal heating, weak MHD turbulence, and scaling laws, Astrophys. 
J., 657, L47-L51, 2007.

Rappazzo, A. F., Velli, M., Einaudi, G., and Dahlburg, R. B.: Nonlinear dynamics of the Parker scenario for coronal heating, Astrophys. J., 677, 1348-1366, 2008.

Regnier, S., Parnell, C. E., and Haynes, A. L.: A new view of quietSun topology from Hinode/SOT, Astron. Astrophys., L47-L50, 2008.

Roberts, D. A., Goldstein, M. L., Klein, L. W., and Matthaeus, W. H.: Origin and evolution of fluctuations in the solar wind: Helios observations and Helios-Voyager comparison, J. Geoph. Res., 92, 12023-12035, 1987.

Sagaut, P. and Cambon, C.: Homogeneous Turbulence Dynamics, Cambridge University Press, 2008.

Sahraoui, F., Belmont, G., and Rezeau, L.: Hamiltonian canonical formulation of Hall MHD: toward an application to weak turbulence, Phys. Plasmas, 10, 1325-1337, 2003

Sahraoui, F., Belmont, G., Rezeau, L., Cornilleau-Wehrlin, N., Pinçon, J. L., and Balogh, A.: Anisotropic turbulent spectra in the terrestrial magnetosheath as seen by the Cluster spacecraft, Phys. Rev. Lett., 96, 075002, doi:10.1103/PhysRevLett.96.075002, 2006.

Sahraoui, F., Galtier, S., and Belmont, G.: Incompressible Hall MHD waves, J. Plasma Phys., 73, 723-730, 2007.

Scalo, J. and Elmegreen, B. G.: Interstellar turbulence II: implications and effects, Annu. Rev. Astron. Astrophys., 42, 275-316, 2004.

Schekochihin, A. A., Cowley, S. C., Dorland, W., Hammett, G. W., Howes, G. G., Quataert, E., and Tatsuno, T.: Astrophysical gyrokinetics: kinetic and fluid turbulence cascades in magnetized weakly collisional plasmas, Astrophys. J. Sup. Series, submitted, 2008.

Shaikh, D. and Zank, G.: Anisotropic cascades in interstellar medium turbulence, Astrophys. J., 656, L17-L20, 2007.
Shebalin, J. V., Matthaeus, W. H., and Montgomery, D.: Anisotropy in MHD turbulence due to a mean magnetic field, J. Plasma Phys., 29, 525-547, 1983.

Smith, C. W., Hamilton, K., Vasquez, B. J., and Leamon, R. J.: Dependence of the dissipation range spectrum of interplanetary magnetic fluctuations on the rate of energy cascade, Astrophys. J., 645, L85-L88, 2006.

Sorriso-Valvo, L., Marino, R., Carbone, V., Noullez, N., Lepreti, F., Veltri, P., Bruno, R., Bavassano, B., and Pietropaolo, E.: Observation of inertial energy cascade in interplanetary space plasma, Phys. Rev. Lett., 99, 115001, doi:10.1103/PhysRevLett.99.115001, 2007.

Sridhar, S. and Goldreich, P.: Toward a theory of interstellar turbulence 1. Weak alfvénic turbulence, Astrophys. J., 432, 612-621, 1994.

Stawicki, O., Gary, P. S., and Li, H.: Solar wind magnetic fluctuations spectra: dispersion versus damping, J. Geoph. Res., 106, 8273-8281, 2001.

Verma, M. K.: Statistical theory of magnetohydrodynamic turbulence: recent results, Phys. Rep., 401, 229-380, 2004.

Warren, H. P.: Multithread hydrodynamic modeling of a solar flare Astrophys. J., 637, 522-530, 2006.

Yamada, M.: Progress in understanding magnetic reconnection in laboratory and space astrophysical plasmas, Phys. Plasmas, 14, 1-16, doi:10.1063/1.2740595, 2007.

Zakharov, V. E., and Filonenko, N. N.: The energy spectrum for stochastic oscillations of a fluid surface, Doclady Akad. Nauk. SSSR, 170, 1292-1295, 1966 [Sov. Phys. Docl., 11, 881-884, 1967].

Zakharov, V. E.: On the spectrum of turbulence in plasma without magnetic field, J. Exp. Theor. Phys., 24, 455-459, 1967.

Zakharov, V. E., L'vov, V., and Falkovich, G. E.: Kolmogorov Spectra of Turbulence I: Wave Turbulence, Springer-Verlag, Berlin, Germany, 1992. 